\title{
Clinical Study on Surgical Outcomes of Endoscopic Dacryocystorhinostomy (DCR) between Powered and Non-Powered Techniques
}

\author{
Dalli Sekhar Reddy ${ }^{1}$ \\ ${ }^{1}$ Department of Otorhinolaryngology, Mount Zion Medical College, Ezamkulam, Adoor, Kerala, India.
}

ABSTRACT

\section{BACKGROUND}

Dacryocystorhinostomy (DCR) is a bypass operation for tear drainage system which involves removal of bone adjacent to lacrimal sac and incorporating lacrimal sac with nasal mucosae. The operative approaches to lacrimal apparatus are external and endoscopic. In recent times advances in surgical technique and better standing of anatomy have made several changes in endoscopic DCR all aiming to improve results, reduce complications, and reduce operative time. Both costly powered and cost affective non-powered instruments are commonly used to make bone windows in endoscopic DCR. The aim of this study is to compare merits and demerits of powered instrument (Drill) and non-powered (Kerrison's punch) DCR.

\section{METHODS}

A randomized comparative study of 60 patients in the age group of 21-70 yrs. who attended our outpatient department regularly and underwent endoscopic DCR procedure at our institution from June 2017 until Aug. 2019 was conducted. Patients were categorised into two groups of 30 patients each, one group which had patients who were operated conventionally by Kerrison's punch and the group with patients who were operated by powered drill. Operative technique, surgical outcome and complications were compared between the two groups.

\section{RESULTS}

A total of 60 endoscopic endonasal DCRs were performed during the period. Male: female ratio; 1:5. $36(60 \%)$ patients presented with watering of eye. Procedure success rate among Kerrison's punch group was $93.33 \%$ vs. $90 \%$ in powered drill group ( $\mathrm{p}=0.476)$. The complications rate in Kerrison's punch group was $10 \%$ compared $20 \%$ in powered drill group $(p=0.032)$. The mean operating time among Kerrison punch group was 33.3 min which was significantly lower than that of powered drill group which was $78.3 \mathrm{~min}$.

\section{CONCLUSIONS}

Endoscopic DCR is keyhole minimal invasive magic surgery. In a comparative study, Kerrison's punch was found to be better tool for making bony window in terms of cost, operating time and complications when compared to powered drill. In terms of success rate of surgery and other factors, there was no significant difference between the two groups.

\section{KEY WORDS}

Dacryocystorhinostomy, Epiphora, Kerrison's Punch, Powered Drill, DCR

\author{
Corresponding Author: \\ Dr. Dalli Sekhar Reddy, \\ \#20-194 Doctor's Colony, \\ Miryalguda-508207, \\ Telangana, India. \\ E-mail: sekharent16@gmail.com
}

DOI: $10.14260 / j e m d s / 2020 / 438$

How to Cite This Article:

Reddy DS. Clinical study on surgical outcomes of endoscopic dacryocystorhinostomy (dcr) between powered and non-powered techniques. J. Evolution Med. Dent. Sci. 2020;9(28): 2012-2015, DOI:

10.14260/jemds/2020/438

Submission 11-04-2020,

Peer Review 05-06-2020,

Acceptance 12-06-2020,

Published 13-07-2020.

Copyright (c) 2020 JEMDS. This is an open access article distributed under Creative Commons Attribution License [Attribution 4.0 International (CC BY 4.0)] 


\section{BACKGROUND}

Dacryocystorhinostomy (DCR) is a procedure of choice for nasolacrimal duct (NLD) obstruction. The earliest operation that would resemble a modern external DCR was attempted by Woolhouse in England in the 18th century. He advocated complete removal of the sac, perforating the lacrimal bone and placing a drain made of gold, lead or silver. Toti in 1904 published what is considered the first modern description of external DCR, incision was made, the periosteum and the sac were elevated, a rhinostomy was created using a punch authors reported success rates of $70-85 \%) .{ }^{1}$ Because of significant bleeding from angular vessels during external DCR various changes in the approach occurred throughout $20^{\text {th }}$ century like change in incisions site, placement of stenting materials, suturing of flaps, use of chisels, rongeurs. Initially, it was believed that external DCR had a higher success rate 85-100\%.

Endoscopic DCR was first demonstrated by Caldwell in 1983. Complete description of Endoscopic transnasal dacryocystorhinostomy was first described in 1989 by Mc Donogh and Meiring. ${ }^{2}$ PJ Wolmard was the person who introduced power instruments endoscopic DCR. Endoscopic DCR is gained popularity in recent years main reason for this from surgeons point of view is availability of high definition cameras and scopes and from patients point of view is better aesthetic with no scar as most of them are female patients, faster return to normal daily activities, higher levels of patient's satisfaction and lower postoperative morbidity. Studies have shown that endoscopic DCR to be more effective in preserving the lacrimal pump system as there is minimal collateral damage to skin, medial canthal tendon muscle and surrounding structures. With the advancement of technology endoscopic dacryocystorhinostomy has become the procedure of choice for otolaryngologists

Epiphora, crusting and swelling in medial canthus region are common annoying symptoms, embarrassing to the patient both socially and functionally and may even endanger the eye. ${ }^{3} \mathrm{~A}$ eye watering due to nasolacrimal duct block is a common clinical problem caused nasolacrimal duct obstruction and is believed to occur due to chronic inflammation of lacrimal sac and lacrimal duct resulting in fibrosis and closure of drainage pathway4. This can be managed surgically by dacryocystorhinostomy (DCR), which bypasses the obstructive lesion and restores the tear flow. Dacryocystitis more common in females of age group 40-50 yrs., reason being smaller dimensions of lacrimal draining pathway and hormonal changes causes more rapid deepithelialisation in sac which contribute for blockage. Clinically two tests which conform site of lesion in lacrimal drainage pathway are Jones dye test and lacrimal probing test. In Jones test 2 drops of $2 \%$ fluorescein dye instilled in the conjunctival sac cotton bud placed in inferior meatus, bud inspected after 5 minutes, bud stained means positive test indicating patent passage, no staining means negative test then we have to do Jones test 2 ,in this with $2 \mathrm{ml}$ normal saline lacrimal syringing done if water comes to inferior meatus it indicate lacrimal pump failure, negative test indicate mechanical block in NLD, if clear fluid comes from same punctum it indicates canalicular block, if clear fluid comes from upper puncta the obstruction is likely at the level of the common canaliculus.
In lacrimal Probing test A small (00) probe should be used initially through lower canaliculi, followed by progressively larger probes. If a "hard stop" is felt during probing of the canaliculi, this implies that the probe has come up against the lacrimal bone, which suggests that the lacrimal drainage system is patent up to the lacrimal sac a "soft stop" is felt; this implies that the probe's distal progress is impeded by soft tissue, suggesting either stenosis or obstruction of the canalicular system. Conjunctivodacryocystorhinostomy is procedure for canaliculi block routine DCR is not of much use. Finally, function of the lacrimal drainage system can be assessed with a dacryocystogram (DCG), in which a radiopaque dye is injected into the lacrimal sac. A magnetic resonance DCG can help to better delineate morphological and functional aspect of lacrimal drainage pathway. DCG is mainly useful in epiphoras which are secondary to maxilla facial trauma. Several techniques, tools such as silastic stunt, Kerrison punch, powered drill, and lasers have been described in endoscopic DCR with the aim of improving operative technique and success rate. Both Kerrison punch and powered drill are widely used in endoscopic DCR. ${ }^{5}$ In this study our objective is to compare the merits and demerits of these two tools in making bony window and try to clarify the superiority of one technique over the other.

\section{METHODS}

After obtaining approval from the Ethical Committee of Mount Zion Medical College, Ezamkulum, Adoor, Pathanamthitta (Dist.) Kerala-691556, a randomized comparative study of 60 patients of either sex aged between 21-70 yrs., who attended our outpatient department regularly complaining of epiphora, discharge from the eye, crusting swelling in the medial canthus of the eye and obstruction to the flow of water on syringing, was conducted. Table 3 shows the demographic data. In all cases, Jones test and Lacrimal probing test done site of lesion or block conformed. All the cases fulfilling the inclusion and exclusion criteria were selected for the study. Every patient was informed about the technique and written consent was also collected from every patient before surgery. 30 patients were selected for Kerrison's punch and 30 for powered drill technique selection done by lottery system. Randomization was done in two study groups by block randomization method with block size 10.60 patients regularly attending ENT Department of Mount Zion Medical College, Ezhamkulam, Adoor Pathanamthitta (Dist.) Kerala-691556, having symptoms of Watering of eye were studied.

\section{Sample Size Calculation}

To examine the Surgical Outcomes of Endoscopic Endonasal Dacryocystorhinostomy (DCR) between powered and nonpowered technique, 60 (30 per group) patients are required to have a $90 \%$ chance of detecting, as significant at the $5 \%$ level, an anticipated increase in the mean operating time (Min) from 55.1 in the I group to 68.3 in the II group with anticipated SD as $15.7 \mathrm{~min}$. Calculation is based on the formula- 


$$
n=\frac{\mathrm{f}(\alpha / 2, \beta) \times 2 \times \sigma^{2}}{\left(\mu 1-\mu^{2}\right)^{2}}
$$

Where,

$\mu 1$ and $\mu_{2}$ are the mean outcomez in the study groups respectively, and

$\sigma$ is the standard deviation.

\section{Surgical Technique}

In all patients preoperative apart from general local examination, eye syringing and, endoscopic evaluation of nose was done. Under aseptic conditions the patient with preanaesthetics using atropine, Phenergan and pentazocine intramuscular before surgery. Nose was packed with $4 \%$ xylocaine and adrenaline as surface anaesthesia. ${ }^{6} 2 \%$ Lidocaine was given to structures anterior to middle turbinate on the lateral nasal wall. Mucosal incision was given, and flap elevated for making bony window on the frontal process of maxillae, two different instruments were used. In the first group, the $2 \mathrm{~mm}$ diamond tip burr drill was used; while in the second group the Kerrison's punch was utilized to get sufficient exposure of the lacrimal sac. In the Initial step to expose lacrimal duct in both groups punch was used once duct is exposed remaining osteotomy is completed either with the drill or the Kerrison's, no instrument conversion was allowed after this. Operating time represents the duration from lateral nasal mucosal incision till the nasal flaps are secured. Postoperatively, outpatient standardized follow up were scheduled at one week then 15 days, then 1,3 , $6, \& 12$ months. Success of the operation was based on Three criteria: the patient subjective improvement of the eye watering, a free flow upon of water on eye syringing test, and patent fistula during endoscopic examination.

\section{Inclusion Criteria}

All the new cases of chronic dacryocystitis coming to outpatient department, who were healthy and nonimmunocompromised.

\section{Exclusion Criteria}

Revision cases, Patients with any intraorbital (or) sinonasal tumours were excluded from the study.

\section{Statistical Methods}

All characteristics were summarized descriptively. For continuous variables, the summary statistics of mean \pm standard deviation (SD) were used. For categorical data, the number and percentage were used in the data summaries and diagrammatic presentation. Chi-square $\left(\chi^{2}\right)$ test was used for association between two categorical variables. The difference of the means of analysis variables between two independent groups was tested by unpaired $t$ test. If the $p$-value was < 0.05 , then the results were considered to be statistically significant otherwise it was considered as not statistically significant. Data were analysed using SPSS software v.23.0. And Microsoft office 2007.

\section{RESULTS}

In our study of 60 cases, following were clinical presentations $36(60 \%)$ cases belongs to epiphora, 12 (20\%) epiphora with swelling, 6 (10\%) epiphora with discharge, 6 (10\%) with epiphora and swelling and discharge. see table 1.

All 60 patients were local residents without any significant racial difference. Fifty patients were women and ten were men, with a mean age of 47 years (Range 21-70 years). 22 cases DCR done in the right eye and 32 in the left eye. See table 3 for demographic data.

The success rate for the powered drill group was $90 \%$, compared with $93.33 \%$ for the Kerrison punch group. The mean operating time of surgery in the powered drill group was $78 \mathrm{~min}$ compared to $33 \mathrm{~min}$ in the Kerrison punch group. See Table 4.

Reported intraoperative and postoperative complications were all minor and included: intranasal synechiae, eye bruise nostril burns and granulations. The overall minor complication rate was $30 \%$ since sample size is small values are significant and there was no record of any major complications. Comparing the two groups it was statistically significant $(\mathrm{p}=0.032)$. See Table 2 . If the $\mathrm{p}$-value was $<0.05$, then the results were considered to be statistically significant otherwise it was considered as not statistically significant.

\begin{tabular}{|c|c|c|c|c|c|}
\hline \multicolumn{3}{|c|}{ Clinical Features } & No. of Cases & \multicolumn{2}{|c|}{ Percentage } \\
\hline \multicolumn{3}{|c|}{ Watering } & 36 & \multicolumn{2}{|c|}{$60 \%$} \\
\hline \multicolumn{3}{|c|}{ Eye watering with swelling } & 12 & \multicolumn{2}{|c|}{$20 \%$} \\
\hline \multicolumn{3}{|c|}{ Watering with discharge } & 6 & \multicolumn{2}{|c|}{$10 \%$} \\
\hline \multicolumn{3}{|c|}{ Watering with swelling and discharge } & 6 & \multicolumn{2}{|c|}{$10 \%$} \\
\hline \multicolumn{3}{|c|}{ Total } & 60 & \multicolumn{2}{|c|}{100} \\
\hline \multicolumn{6}{|c|}{ Table 1. Presenting Complaints } \\
\hline \multirow{2}{*}{$\begin{array}{c}\text { Minor } \\
\text { Complications }\end{array}$} & \multicolumn{2}{|c|}{$\begin{array}{c}\text { Drill } \\
\mathrm{N}=30\end{array}$} & \multicolumn{2}{|c|}{$\begin{array}{l}\text { Kerrison's Punch } \\
\qquad \mathrm{N}=\mathbf{3 0}\end{array}$} & \multirow{2}{*}{$\begin{array}{c}\text { P } \\
\text { Value }\end{array}$} \\
\hline & $\mathbf{n}$ & $\%$ & $\mathbf{n}$ & $\%$ & \\
\hline Intra nasal synechiae & 1 & $3.3 \%$ & 2 & $6.7 \%$ & \multirow{5}{*}{$\begin{array}{c}0.032 \\
\text { (sig) }\end{array}$} \\
\hline Eye bruise & 2 & $6.7 \%$ & 1 & $3.3 \%$ & \\
\hline Nostril burn & 2 & $6.7 \%$ & 0 & $0.0 \%$ & \\
\hline Granulation & 1 & $3.3 \%$ & 0 & $0.0 \%$ & \\
\hline Total & 6 & $20.0 \%$ & 3 & $10.0 \%$ & \\
\hline \multicolumn{6}{|c|}{ Table 2. Minor Complication of Endoscopic DCR } \\
\hline
\end{tabular}

\begin{tabular}{|c|c|c|c|c|c|c|c|}
\hline \multirow{2}{*}{\multicolumn{4}{|c|}{ Overall }} & \multicolumn{4}{|c|}{ Instrument used } \\
\hline & & & & & & & unch \\
\hline \multirow{2}{*}{ Age (years) } & Range & \multicolumn{2}{|c|}{$21-70$} & \multicolumn{2}{|c|}{$25-60$} & \multicolumn{2}{|c|}{$21-70$} \\
\hline & Mean \pm SD & \multicolumn{2}{|c|}{$47.1 \pm 5.3$} & \multicolumn{2}{|c|}{$45.5 \pm 4.3$} & \multicolumn{2}{|c|}{$49.3 \pm 6.7$} \\
\hline \multirow{2}{*}{ Gender } & Male & 10 & $16.6 \%$ & 4 & $13.79 \%$ & 6 & $19.3 \%$ \\
\hline & Female & 50 & $83.4 \%$ & 25 & $86.20 \%$ & 25 & $80.64 \%$ \\
\hline \multirow{2}{*}{ Eye affected } & Right & 22 & $36.6 \%$ & 10 & $33.33 \%$ & 12 & $40 \%$ \\
\hline & Left & 38 & $63.3 \%$ & 20 & $66.66 \%$ & 18 & $60 \%$ \\
\hline Follow up & Range & \multicolumn{2}{|c|}{$2-12$} & \multicolumn{2}{|c|}{$3-12$} & \multicolumn{2}{|r|}{$2-8$} \\
\hline \multicolumn{8}{|c|}{ Table 3. Demographic Statistics } \\
\hline
\end{tabular}

\begin{tabular}{|c|c|c|c|c|c|}
\hline \multicolumn{2}{|c|}{ Parameter } & $\begin{array}{l}\text { Overall } \\
(N=60)\end{array}$ & $\begin{array}{c}\text { Powered } \\
\text { Drill } \\
(\mathrm{N}=30)\end{array}$ & $\begin{array}{c}\text { Kerrison's } \\
\text { Punch } \\
(\mathrm{N}=30)\end{array}$ & $\begin{array}{c}P \\
\text { Value }\end{array}$ \\
\hline \multicolumn{2}{|c|}{ Success Rate } & $91.66 \%$ & $90 \%$ & $93.33 \%$ & \multirow{2}{*}{0.476} \\
\hline Operating & Range & $30-100$ & $45-100$ & $30-45$ & \\
\hline Time & Mean + SD & $56.1+4.7$ & $78.3+5.6$ & $33.3+4.1$ & $<0.001$ (sig) \\
\hline \multicolumn{6}{|c|}{ Table 4. Procedure Success and Operating Time } \\
\hline
\end{tabular}

\section{DISCUSSION}

The external DCR is standard treatment and endoscopic DCR is minimal invasive procedure. Endoscopic DCR is gaining popularity in recent years main reason for this from 
surgeons' point of view is availability of high definition cameras and scopes and from patients' point of view is better aesthetic with no scar as most of them are female patients 7 . External DCR or endonasal DCR Most important step is making bony window on lateral wall of nose (frontal process of maxilla). Presently various techniques are available for bony window in endo DCR like conventional punch, powered drill, and diode laser. Each of gadgets used has various merits and demerits. The value of non-traumatic procedure is an emerging concept in endoscopic DCR. Without drill we can do DCR surgery but without punch it is not possible to complete surgery. Creation of a large bony window by itself does not mean successful procedure other factor which contribute for success are intra operative tissue damage, bleeding and duration of surgery because of this idea concept of cold instruments and tools are gaining popularity.8,9 The main idea of our study is to show which technique is safe, has least complications and high success rate. When high speed drill is used it generate temperature around $75^{0}$ at site of drill which causes local tissue damage and this is responsible for postoperative scarring, synechiae and failure ${ }^{10}$. Powered drill usage in nose need surgical skills in in experienced hands their high risk of damage to lamina papyracea, middle turbinate, accidental opening of agar cell which is also shown in literatere ${ }^{11}$. With powered drill some amount of bone dust is generated this is one of the reasons for failure as it contributes for synechiae and granulations In our current study procedure success rate among Kerrison punch group was $93.33 \%$ vs $90.00 \%$ in the powered drill group $(\mathrm{p}=0.476)$.See Table 4.

Operating time is a valuable factor in health care economics less operating time may accomplish increased surgical efficiency and reduction morbidity. ${ }^{12}$ Powered drill need more time for setup, irrigation during drilling and suctioning after that to remove generated bony dust, with meticulous use to prevent any injure to surrounding vital structures. Our study has shown that with usage of punch has less preoperative bleeding compared to drill the reason may be that minor vessels get crushed with punch whereas with drill bleeding needs additional bipolar cautery to control. Our results showed that there is a statistically significant difference operating time for endoscopic DCR using the drill 78 min compared with Kerrison punch $33 \mathrm{~min}$ p-value $<0.001$. Our experience has shown that patient with facial injury in past and who present with eye watering now in such cases powder drill use is very difficult as whole of nasal framework moves drilling. In such cases Kerrison punch is only option Our overall rate of minor complication synechiae, bleeding bruise (30\%) is not much statistical different from previous studies on endoscopic DCR. Rahman et al. reported a minor complication rate of $28 \% .13$ Despite meticulous work with the drill, we observed two cases with minor nostril burn indicating a requirement of more carefulness in handling the drill. ${ }^{14}$ The limitations of this study are mainly the retrospective design of the study and the moderately small sample size.

\section{CONCLUSIONS}

Endoscopic DCR is keyhole minimal invasive magic surgery in comparison to external DCR. In this study, Kerrison's punch was found to be a better tool for making bony window in terms of cost, operating time, and complications, when compared to powered drill. In terms of success rate of surgery and other factors, not much difference was found between the two groups. Every ENT surgeon should know the pros and cons of both instruments.

\section{REFERENCES}

[1] Chandler PA. Dacryocystorhinostomy. Trans Am Ophthalmol Soc 1936;34:240-63.

[2] McDonogh M, Meiring JH. Endoscopic transnasal dacryocystorhinostomy. J Laryngol Otol 1989;103 (6):585-7.

[3] Jacobs HB. Symptomatic epiphora. Br J Ophthalmology 1959;43 (7):415-34.

[4] Önerci M. Dacryocystorhinostomy: diagnosis and treatment of nasolacrimal canal obstructions. Rhinology 2002;40 (2):49-65.

[5] Wormald PJ, Tsirbas A. Powered endoscopic dacryocystorhinostomy. The Lacrimal System 2006: p. 223-35.

[6] Tripathi A, Lesser THJ, O’Donnell NP, et al. Local anesthetic endonasal endoscopic laser dacryocystorhinostomy: analysis of patient's acceptability and various factors affecting the success of this procedure. Eye (Lond) 2002;16 (2):146-9.

[7] Wormald PJ. Powered endonasal dacryocystorhinostomy. Laryngoscope 2002;112 (1):6972.

[8] Lindbergh JV, Anderson RL, Bumsted RM, et al. Study of intranasal ostium external dacryocystorhinostomy. Arch Ophthalmol 1982;100 (11):1758-62.

[9] Kim SY, Paik JS, Jung SK, et al. No thermal tool using methods in endoscopic dacryocystorhinostomy: no cautery, no drill, illuminator, no more tears. Eur Arch Otorhinolaryngol 2013;270 (10):2677-82.

[10] Codère F, Denton P, Corona J. Endonasal dacryocystorhinostomy: a modified technique with preservation of the nasal and lacrimal mucosa. Ophthalmic Plast Reconstr Surg 2010;26 (3):161-4.

[11] Badilla J, Dolman PJ. Cerebrospinal fluid leaks complicating orbital or oculoplastic surgery. Arch Ophthalmol 2007;125 (12):1631-4.

[12] Jin HR, Yeon JY, Choi MY. Endoscopic dacryocystorhinostomy: creation of a large marsupialized lacrimal sac. J Korean Med Sci 2006;21(4):719-23.

[13] Tsai M. The true cost of operating room time. Arch Surg 2011;146 (7):886.

[14] Rahman SH, Tarafder KH, Ahmed MS, et al. Endoscopic dacryocystorhinostomy. Mymensingh Med J 2011;20 (1):28-32. 